\title{
Chronic Active Hepatitis B with COVID-19 in Pregnancy: A Case Report
}

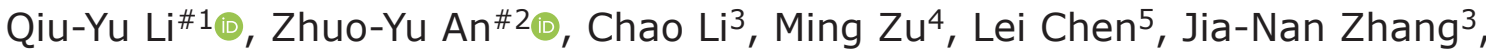 \\ Yang-Yu Zhao ${ }^{6}$, Ning Shen ${ }^{1}$ and Qing-Gang Ge ${ }^{* 3}$
}

${ }^{1}$ Department of Respiratory and Critical Care Medicine, Peking University Third Hospital, Beijing, China; ${ }^{2}$ Peking University Institute of Hematology, Peking University People's Hospital, Beijing, China; ${ }^{3}$ Department of Critical Care Medicine, Peking University Third Hospital, Beijing, China; ${ }^{4}$ Department of Gastroenterology, Peking University Third Hospital, Beijing, China; ${ }^{5}$ Department of Respiratory and Critical Care Medicine, Tongji Hospital, Tongji Medical College, Huazhong University of Science and Technology, Wuhan, Hubei, China; ${ }^{6}$ Department of Obstetrics and Gynecology, Peking University

Third Hospital, Beijing, China

\begin{abstract}
Currently, infection with coronavirus disease 2019 (COVID-19), caused by severe acute respiratory syndrome coronavirus 2 (SARS-CoV-2), during pregnancy is a problem worthy of attention, especially in patients with underlying diseases. In this case report, we present a case of chronic active hepatitis B with COVID-19 in pregnancy. A 31-yearold woman at 29 weeks of gestation who had a history of chronic hepatitis B virus infection discontinued antiviral treatment, was admitted to the hospital with chronic active hepatitis B, and tested positive for SARS-CoV-2 infection. In this case, we applied liver protective and antiviral agents, and low-dose dexamethasone therapy to successfully treat the critically ill pregnant woman suffering from chronic active hepatitis B combined with COVID-19.
\end{abstract}

Citation of this article: Li QY, An ZY, Li C, Zu M, Chen L, Zhang JN, et al. Chronic active hepatitis B with COVID-19 in pregnancy: A case report. J Clin Transl Hepatol 2021; 000(000):000-000. doi: 10.14218/JCTH.2020.00085.

\section{Introduction}

At present, the severe acute respiratory syndrome coronavirus-2 (SARS-CoV-2) caused the epidemic of coronavirus disease 2019 (COVID-19). ${ }^{1}$ The World Health Organization has declared the COVID-19 outbreak as a pandemic on March 11, 2020. ${ }^{2}$ With the expansion of the infected population, the clinical characteristics, management and prognosis of pregnant women with COVID-19 have received more and more attention, especially in the presence of underlying diseases. Elevated bilirubin and transaminase are common in patients with COVID-19,3,4 suggesting that liver damage is often accompanied with SARS-2 infection. As

Keywords: COVID-19; SARS-CoV-2; Pregnancy; Active hepatitis B. Abbreviations: COVID-19, coronavirus disease 2019; HBV, hepatitis B virus; SARS-CoV-2, severe acute respiratory syndrome coronavirus 2. Received: 08 September 2020; Revised: 13 November 2020; Accepted: 28 December 2020

\#These authors contributed equally to this work.

*Correspondence to: Qing-Gang Ge, Department of Critical Care Medicine, Peking University Third Hospital, Beijing 100191, China. Tel: +86-10-82265530, Fax: +86-10-82074373, E-mail: qingganggelin@126.com for SARS-CoV-2 and HBV co-infected patients, it has also been reported that the proportion of abnormal liver function tests is similar to SARS-CoV-2 alone infection. ${ }^{5-7}$ In this case, there has been no sufficient evidence on treatment for such patients, such as whether the use of systematic glucocorticoids will slow down virus clearance, etc., especially for pregnant women. This case reports the successful treatment of antiviral, liver protection and low-dose dexamethasone to effectively treat acute chronic hepatitis B with COVID-19 in pregnancy.

\section{Case report}

A 31-year-old woman at 29 weeks of gestation presented to the hospital on March 29, 2020, with a 30-day history of malaise, diarrhea, itching, and jaundice. She also reported having experienced exertional dyspnea with cough. A nucleic acid test for SARS-CoV-2 was thus performed prior to admission, and yielded positive result.

The patient had a history of chronic hepatitis $B$ virus (HBV) infection, for 9 years. She reported a transaminase level of nearly $1,000 \mathrm{U} / \mathrm{L}$ in September 2019. She had taken "tenofovir" once a day, which she had discontinued (by herself) after pregnancy (September 2020).

The vital signs on admission were as follows: $\mathrm{SpO}_{2} 96 \%$ (no oxygen inhalation therapy); heart rate of $102 \mathrm{bpm}$; blood pressure of $119 / 87 \mathrm{mmHg}$; respiratory rate of 20 breaths per minute; and, temperature of $36.3^{\circ} \mathrm{C}$. No swelling of the lower limbs was observed. Nasopharyngeal swabs tested positive for SARS-CoV-2 nucleic acid. Chest and abdominal computed tomography showed no obvious lesions, except for a plump pancreas.

The laboratory examination was expanded upon after admission. Blood examination showed white blood cell count of $13.06 \times 10^{9} / \mathrm{L}$, normal platelet count $\left(258 \times 10^{9} / \mathrm{L}\right)$, and HBV-DNA of $2.56 * 10^{5} / \mathrm{mL}$. Coagulation test showed an international normalized ratio of 1.33 , an activated partial thromboplastin time of $34.3 \mathrm{~s}$, a fibrinogen level of 3.17 $\mathrm{g} / \mathrm{L}$ and D-dimer level of $0.94 \mu \mathrm{g} / \mathrm{mL}$ (Table 1 ). Obstetric ultrasound showed a single live fetus, equivalent to $29+2$ weeks, a slightly faster fetal heart rate, placenta grade I, and low placenta.

The patient was diagnosed with the following: 1 . COVID-19, mild type; 2. 29 weeks of gestation, G3P1, head position; and, 3. Chronic active hepatitis $B$ with liver function abnormality (Child grade B).

After admission, the patient was administered adenosyl- 
Table 1. Laboratory findings

\begin{tabular}{|c|c|c|c|c|}
\hline & $2020 / 3 / 20$ & $2020 / 3 / 29$ & $2020 / 3 / 30$ & $2020 / 4 / 2$ \\
\hline WBC $\left(* 10^{9} / L\right)$ & ND & ND & 13.06 & 10.27 \\
\hline PIt $\left(* 10^{9} / \mathrm{L}\right)$ & ND & ND & 258 & 274 \\
\hline AST (IU/L) & 795 & 1,321 & 506 & 55 \\
\hline ALT (IU/L) & 503 & 858 & 534 & 147 \\
\hline D-Dimer $(\mu \mathrm{g} / \mathrm{mL})$ & ND & ND & 0.94 & 0.72 \\
\hline T-Bil $(\mu \mathrm{mol} / \mathrm{L})$ & 183.1 & 117.8 & 105.3 & 45.0 \\
\hline D-Bil $(\mu \mathrm{mol} / L)$ & ND & ND & 101.3 & 41.8 \\
\hline PT (s) & ND & ND & 16.6 & 14.0 \\
\hline APTT (s) & ND & ND & 34.3 & 29.9 \\
\hline INR & ND & ND & 1.33 & 1.07 \\
\hline HBV-DNA & ND & ND & $2.56 * 10^{5} / \mathrm{mL}$ & $1.67 * 10^{3} / \mathrm{mL}$ \\
\hline $\mathrm{HCV}-\mathrm{Ab}$ & ND & ND & Negative & ND \\
\hline $\mathrm{HBs}-\mathrm{Ag}$ & ND & ND & Positive & ND \\
\hline HBs-Ab & ND & ND & Negative & ND \\
\hline $\mathrm{HBe}-\mathrm{Ag}$ & ND & ND & Positive & ND \\
\hline $\mathrm{HBe}-\mathrm{Ab}$ & ND & ND & Negative & ND \\
\hline $\mathrm{HBc}-\mathrm{Ab}$ & ND & ND & Positive & ND \\
\hline
\end{tabular}

Abbreviations: Ab, antibody; Ag, antigen; ALT, alanine aminotransferase; APTT, activated partial thromboplastin time; AST, aspartate aminotransferase; D-Bil, direct bilirubin; HBc-Ab, hepatitis B virus core antibody; HBs, hepatitis B virus surface protein; HBV, hepatitis B virus; HCV, hepatitis C virus; INR, international normalized ratio; PIt, platelet; PT, prothrombin time; T-Bil, total bilirubin; WBC, white blood cell.

methionine succinate, reduced glutathione to protect the liver, tenofovir (as an antiviral), and a small dose of dexamethasone (10 $\mathrm{mg} \mathrm{q.d}$. for 3 days) to promote fetal lung maturation and reduce systemic inflammation. She was also monitored closely for liver function, coagulation function, and HBV-DNA change in trend (Fig. 1). Considering the results of the fetal ultrasound and fetal heart rate monitoring, we estimated that the fetus was normal and determined that there was no indication for emergency termination of pregnancy.

After treatment, the patient's liver function, and other indicators gradually improved. The patient's respiratory symptoms were also reduced, dyspnea relieved, and $\mathrm{SpO}_{2}$ maintained at a stable high level. After 3 weeks, the SARSCoV-2 nucleic acid test was re-performed twice, and both results were negative. She was discharged from the hospital on May 1, 2020.

We prescribed antiviral treatment with tenofovir and recommended regular follow-up examinations at the outpatient clinic. Throughout, her HBV-DNA level was maintained at about $1.27 * 10^{2} \mathrm{IU} / \mathrm{L}$. She gave birth to a baby boy vaginally

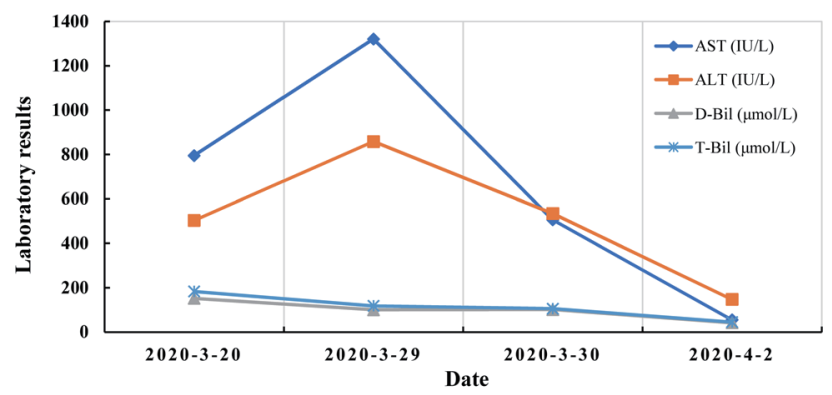

Fig. 1. Changes in laboratory tests for liver function during the infection and management course. at 39 weeks of pregnancy, with $200 \mathrm{~mL}$ of vaginal bleeding. The mother was in good condition and the neonatal Apgar score was 9 and weighed $3,490 \mathrm{~g}$.

\section{Ethics approval and consent to participate}

Informed consent for the collection of medical history and blood samples was obtained in compliance with the Declaration of Helsinki and approved by the local ethical committee (Peking University Third Hospital, Medical Science Research Ethical Committee Approval: IRB00006761-M2020060), and the patient provided informed consent for publication of the case.

\section{Discussion}

This case report described a young woman who was infected with SARS-CoV-2 during pregnancy and who had a history of chronic HBV infection. Pregnant women are prone to liver damage, due to changes in liver structure and function, such as high basal metabolic rate, inactivation of large amounts of estrogen, and vigorous metabolites in the liver. Previous studies have reported that pregnant women with chronic HBV infection who have high alanine aminotransferase or who have been treated for less than 1 year before pregnancy have a higher risk of severe hepatitis after stopping antiviral drugs. ${ }^{8}$ A systematic review of 108 pregnant women from different countries showed that compared with SARS, Middle Eastern respiratory syndrome, influenza $A$, hepatitis $E$, etc., cases of severe illness and death among COVID-19 patients during pregnancy are extremely rare. A review determined that pregnancy causes changes in immunity which are conducive to an anti-inflammatory response and reduces the mortality rate of COVID-19.9,10 
Clinical manifestations of COVID-19 patients during pregnancy are similar to those in non-pregnant patients, mainly including fever, dry cough, and dyspnea. In terms of treatment, since there are no specific antiviral drugs, supportive treatment is still the main method, such as sustained monitoring, maintaining hemodynamics and internal environment stability, and avoiding electrolyte disorders and dehydration. 11,12

Based on existing reports, SARS-CoV-2 has caused few diarrhea cases $(3.8 \%)$. We consider that the patient's diarrhea was mainly caused by hepatic insufficiency. ${ }^{13}$ Differential diagnosis should include acute fatty liver of pregnancy and intrahepatic cholestasis of pregnancy. The main manifestations of the case included jaundice, fatigue, anorexia, and other gastrointestinal symptoms, but there was no hypoglycemia, low fibrin, nor evidence of acute kidney injury. Besides, no obvious hepatic structural lesions were observed by ultrasound, and diagnosis of acute fatty liver of pregnancy could be excluded. The transaminase level was significantly increased, and there was a history of chronic HBV infection, therefore, intrahepatic cholestasis of pregnancy was not fully supported.

There is still controversy about whether glucocorticoids should be used for COVID-19. SARS-CoV-2 has caused an excessively strong inflammation effect in COVID-19 patients, especially in critically ill patients, leading to severe multiorgan damage. Some experts believe that such antiinflammatory effects can be applied to treat such patients. However, glucocorticoids can increase the risk of complications and delay the clearance of the virus. Even short-term use of glucocorticoids may cause venous thrombosis, fractures, sepsis, delayed virus clearance, and other steroidrelated complications. ${ }^{14}$ It should also be noted that the efficacy and safety of glucocorticoids depend on the dosage. The RECOVERY trial conducted in the UK showed that the application of low-dose dexamethasone ( $6 \mathrm{mg}$ q.d.) can reduce 28 -day mortality. ${ }^{15}$ Treatment in this case revealed a small dose of dexamethasone is suitable for a short period to prevent the exacerbation of inflammation in COVID- 19 .

A meta-analysis showed telbivudine, lamivudine, and tenofovir to be safe during pregnancy and to not increase the incidence of adverse outcome events. ${ }^{16}$ In this case, the tenofovir antiviral treatment was promptly resumed based on adequate liver protection treatment, and a better curative effect was obtained. Coagulation dysfunction is more common among COVID-19 patients, especially for liver dysfunction in this case. Therefore, monitoring coagulation function during the treatment to prevent serious complications, such as disseminated intravascular coagulation, and emergency termination of pregnancy is considered when the condition worsens. ${ }^{17}$

In summary, we used a comprehensive treatment paradigm of antiviral, liver protection and low-dose dexamethasone to effectively treat this case of acute chronic hepatitis $B$ with COVID-19 in pregnancy. The patient avoided termination of pregnancy and was well-healed. Pregnant women who meet the indications for viral treatment should receive standardized treatment in time to avoid severe hepatitis. This case shows that low-dose dexamethasone can be used safely and effectively in pregnant women with dual infection of HBV and SARS-CoV-2, to reduce systemic symptoms, which showed no obvious side effects that aggravated either viral infection.

\section{Acknowledgments}

We thank the Medical team members of fighting against COVID-19 of Peking University. We thank Dr. Jie Xu, Dr. Shi-Gang
Ding, and Dr. Dian-Rong Xiu for the scientific discussion.

\section{Funding}

This work was supported by the National Natural Science Foundation of China (Grant No. 81900641).

\section{Conflict of interest}

The authors have no conflict of interests related to this publication.

\section{Author contributions}

Conception, design, and drafting of the manuscript (QYL), manuscript preparation with substantial contributions to intellectual content, critical editing and revisions of the manuscript (ZYA, CL, MZ, LC, NS, YYZ, JNZ, QGG).

\section{References}

[1] CDC. Data on COVID-19 during pregnancy: severity of maternal illness. Available from: https://www.cdc.gov/coronavirus/2019-ncov/cases-updates/special-populations/pregnancy-data-on-covid-19.html.

[2] Patel KP, Patel PA, Vunnam RR, Hewlett AT, Jain R, Jing R, et al. Gastrointestinal, hepatobiliary, and pancreatic manifestations of COVID-19. J Clin Virol 2020;128:104386. doi:10.1016/j.jcv.2020.104386.

[3] Fu Y, Zhu R, Bai T, Han P, He Q, Jing M, et al. Clinical features of patients infected with coronavirus disease 2019 with elevated liver biochemistries: A multicenter, retrospective study. Hepatology 2020. doi:10.1002/hep 31446.

[4] Hao SR, Zhang SY, Lian JS, Jin X, Ye CY, Cai H, et al. Liver enzyme elevation in coronavirus disease 2019: A multicenter, retrospective, cross-sectional study. Am J Gastroenterol 2020;115:1075-1083. doi:10.14309/ajg. 0000000000000717

[5] Zou X, Fang M, Li S, Wu L, Gao B, Gao H, et al. Characteristics of liver function in patients with SARS-CoV-2 and chronic HBV coinfection. Clin function in patients with SARS-CoV-2 and chronic HBV coin
Gastroenterol Hepatol 2020. doi:10.1016/j.cgh.2020.06.017.

[6] Huang C, Wang Y, Li X, Ren L, Zhao J, Hu Y, et al. Clinical features of patients infected with 2019 novel coronavirus in Wuhan, China. Lance 2020;395:497-506. doi:10.1016/S0140-6736(20)30183-5.

[7] Feng G, Zheng KI, Yan QQ, Rios RS, Targher G, Byrne CD, et al. COVID-19 and liver dysfunction: Current insights and emergent therapeutic strategies. J Clin Transl Hepatol 2020;8:18-24. doi:10.14218/JCTH 2020 00018.

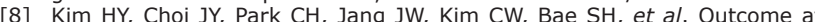
ter discontinuing antiviral agents during pregnancy in women infected ter discontinuing antiviral agents during pregnancy in women infected
with hepatitis B virus. J Clin Virol 2013;56:299-305. doi:10.1016/j.jcv. with hepatitis

[9] Zaigham M, Andersson O. Maternal and perinatal outcomes with COVID-19: A systematic review of 108 pregnancies. Acta Obstet Gynecol Scand 2020 99:823-829. doi:10.1111/aogs.13867.

[10] Berhan Y. What immunological and hormonal protective factors lower the risk of COVID-19 related deaths in pregnant women? J Reprod Immunol 2020;142:103180. doi:10.1016/j.jri.2020.103180.

[11] Yan J, Guo J, Fan C, Juan J, Yu X, Li J, et al. Coronavirus disease 2019 in pregnant women: a report based on 116 cases. Am J Obstet Gynecol 2020:223:111.e1-111.e14. doi:10.1016/j.ajog.2020.04.014.

[12] Covid-19 and pregnancy. BM] 2020;369:m1672. doi:10.1136/bmj.m1672.

[13] Guan WJ, Ni ZY, Hu Y, Liang WH, Ou CQ, He JX, et al. Clinical characteristics of coronavirus disease 2019 in China. N Engl J Med 2020;382:1708-1720. doi:10.1056/NEJMoa2002032.

[14] Waljee AK, Rogers MA, Lin P, Singal AG, Stein JD, Marks RM, et al. Short term use of oral corticosteroids and related harms among adults in the United States: population based cohort study. BMJ 2017;357:j1415. doi:10.1136/ bmj.j1415.

[15] Horby P, Lim WS, Emberson JR, Mafham M, Bell JL, Linsell L, et al. Dexamethasone in hospitalized patients with Covid-19 - Preliminary report. N Engl J Med 2020:NEJMoa2021436, doi:10.1056/NEJMoa2021436.

[16] Brown RS Jr, McMahon BJ, Lok AS, Wong JB, Ahmed AT, Mouchli MA, et al. Antiviral therapy in chronic hepatitis B viral infection during pregnancy A systematic review and meta-analysis. Hepatology 2016;63:319-333. doi: 10.1002/hep.28302.

[17] Levi M, Thachil J, Iba T, Levy JH. Coagulation abnormalities and thrombosis in patients with COVID-19. Lancet Haematol 2020;7:e438-e440. doi:10.1016/S2352-3026(20)30145-9. 